\title{
Healthcare Resource Utilization Domain
}

National Cancer Institute

\section{Source}

National Cancer Institute. Healthcare Resource Utilization Domain. NCI Thesaurus. Code C49589.

A subject domain utilized for the submission of information encompassing and representing data, vocabulary or records related to healthcare resource utilization. 